\title{
Group B Streptococci and-Clindamycin Induced Phenotypic Resistance Pattern in Pregnant Women, Case Study of Sarem Women's Hospital
}

\section{ART ICLE INFO}

\section{Article Type}

Original research

\section{Authors}

Nomanpour B.* $P h D$,

Lashgari P. ${ }^{1} M S c$,

Hasani M. ${ }^{1} B S C$

How to cite this article

Nomanpour B, Lashgari P, Hasa-

ni M. Group B Streptococci and-

Clindamycin Induced Phenotypic

Resistance Pattern in Pregnant

Women, Case Study of Sarem Wo-

men's Hospital. Sarem Journal of Reproductive Medicine. 2019;3(2): :53-57.

\section{A B S T R A C T}

Aims Streptococcus agalactiae strains or group B streptococci (GBS) are the natural flora of the anogenital area of women and are the most important cause of death in the first week of infant birth. The aim of this study was to investigate the isolated strains of group B streptococci and phenotypic resistance pattern of clindamycin induced in pregnant women.

Materials \& Methods This cross sectional-descriptive study was done on vaginal culture of 861 pregnant women. After a physical examination by a gynecologist, two vaginal swabs of vaginal discharges were taken in $1 \mathrm{ml}$ sterile normal saline tube and two slides were delivered to hospital laboratory for staining. The Specimens were cultured on Sheep blood agar, Eosinmethylene blue (EMB) agar and Sabouraud dextrose agar at $35 \pm 1 \dot{C}$ and Chocolate agar were incubated in candle jar at the same temperature. After diagnostic tests, GBS was isolated and microbial susceptibility test was performed. Also, the Erythromycin-inducible resistances to clindamycin was measured by phenotypic D-test for the samples.

Findings Of the 861 samples sent to the laboratory, 141 cases (16.38\%) were positive for GBS. The sensitivity of GBS isolates to penicillin, cefalexin, and ceftazidime was 98.58\%, 97.8\% and $98.88 \%$, respectively. There was no resistance to ampicillin and ceftriaxone in the samples, but $2.84 \%$ of them were resistant to ciprofloxacin. Clindamycin induced resistance was positive in $14.89 \%$ of pregnant women.

Conclusion Among isolated bacteria, 16.38\% of them are part of group B streptococci that $14.89 \%$ of them are clindamycin induced resistance and $38.6 \%$ of them have concurrent resistance to erythromycin and clindamycin.

Keywords Streptococcus agalactiae; Drug resistance; Pregnant women

\section{I T A T I O N L I N K S}

[1] Therapeutic guidelines: antibiotic [2] Group B Streptococci causing neonatal bloodstream infection: antimicrobial susceptibility and serotyping results from SENTRY centers in the Western Hemisphere [3] Carriage of group B Streptococci during pregnancy: a puzzler [4] WHO recommendations on interventions to improve preterm birth outcomes [5] Transmission of group B Streptococci among parturient women and their neonates [6] Prevention of perinatal group B Streptococcal disease: revised guidelines from CDC, 2010 [7] Invasive group B Streptococcus (GBS) disease in Norway 1996-2006 [8] The association of preterm labor with vaginal colonization of group B Streptococci [9] Antibiotic resistance patterns in invasive group B Streptococcal isolates [10] Prevalence and mechanisms of erythromycin resistance in group A and group B Streptococcus: implications for reporting susceptibility results [11] Invasive bacteria isolates from children with severe infections in a Nigerian hospital [12] High rates of erythromycin and clindamycin resistance among OBGYN isolates of group B Streptococcus [13] High burden of group B Streptococcus: an invasive bacterium among pregnant women referring to health centers of Sanandaj, Iran [14] Streptococcus agalactiae in a large Portuguese teaching hospital: antimicrobial susceptibility, serotype distribution, and clonal analysis of macrolide-resistant isolates [15] Prevalence of maternal group B Streptococcal colonisation in European countries [16] Evaluation of group B Streptococci colonization rate in pregnant women and their newborn [17] Martinez de Tejada Weber B. Antibiotic resistance patterns among group B Streptococcus isolates: implications for antibiotic prophylaxis for early-onset neonatal sepsis [18] Colonization rate of group B Streptococcus (GBS) in pregnant women using GBS agar medium [19] Capsular serotype and antibiotic resistance of group B Streptococci isolated from pregnant women in Ardabil, Iran [20] The prevalence of erythromycin resistance in group B Streptococcal isolates at a University Hospital in Taiwan

\section{Article History}

Received: January 10, 2018

Accepted: April 17,2018

ePublished: June 15, 2019 
كند[5]. شايعترين علايم شامل عفونت زودرس، ديسترس تنفسى و عفونت خون هستند[6].

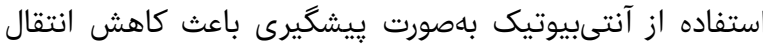

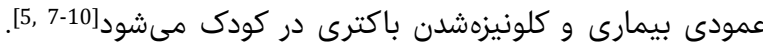

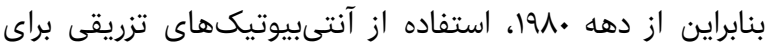

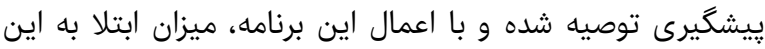

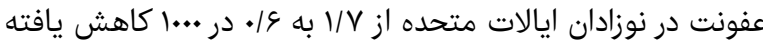

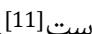

بر اساس دستورالعمل سازمانهاى جهانى، كشت ناحيه ركتال-

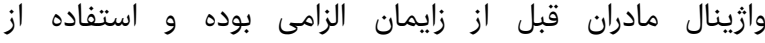

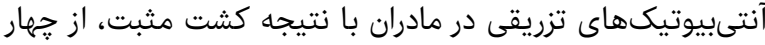

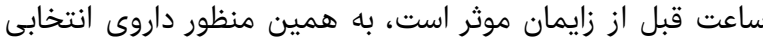

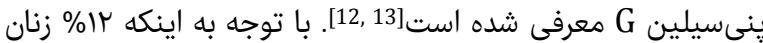

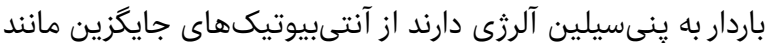

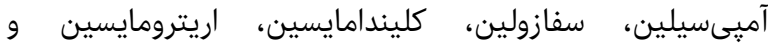

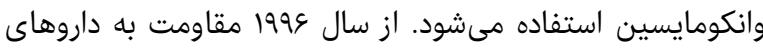

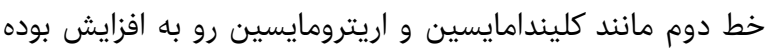

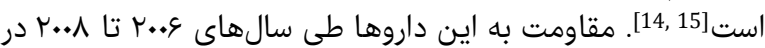

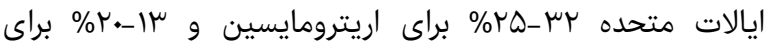

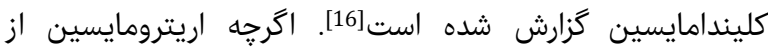

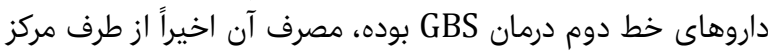

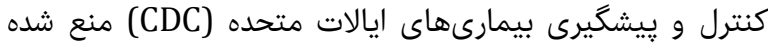

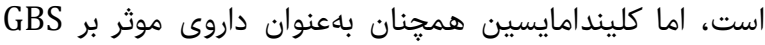

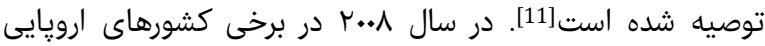

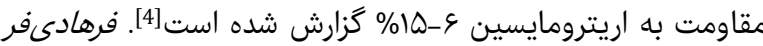

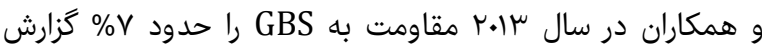

كردهاند [14].

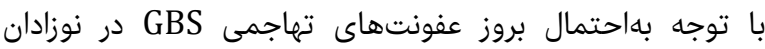

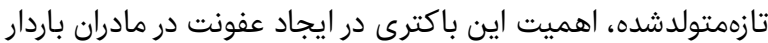

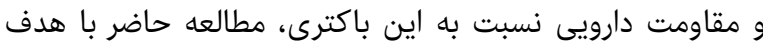

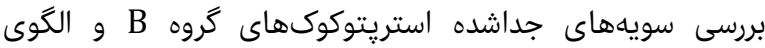

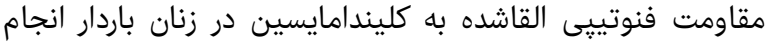

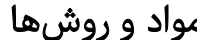

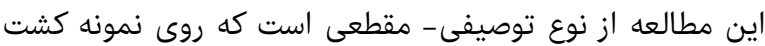

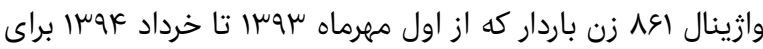

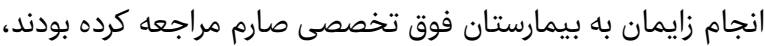

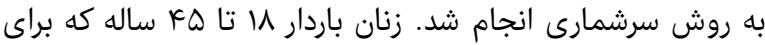

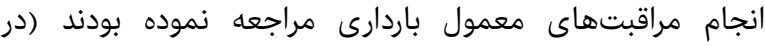

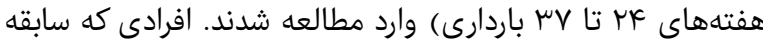

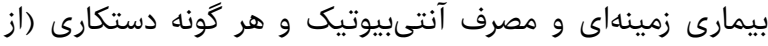
جمله سركلاز) داشتند وارد مطالعه نشدند.

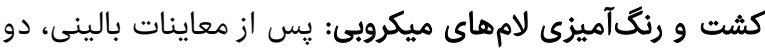

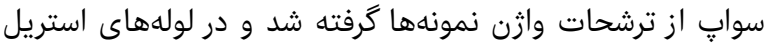

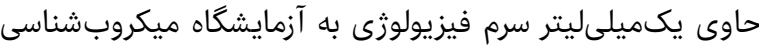

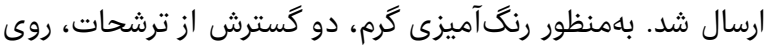

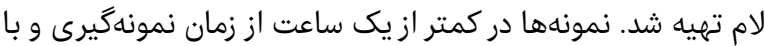

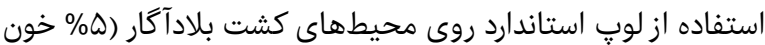

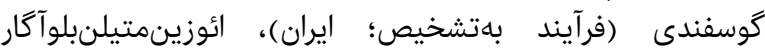
(كنداليده؛ ايران)، شكلاتآكار (فرآيند بهتشخيص؛ ايران)، ايران)، محيط

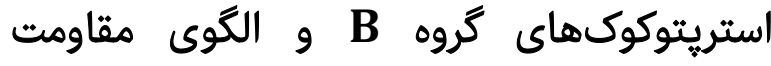
فنوتييى القاشده به كليندامايسين در زنان ماندان باردار،

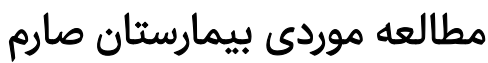

بيزن نعمانيور" PhD بخش ميكروبشناسى، مركز تحقيقات بارورى و نابارورى صارم، بيمارستان

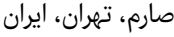
MSc بيمان لشكرى تيكران ايران بخش ميكروبشناسى، مركز تحقيقات بارورى و نابارورى صارم، بيمارستان

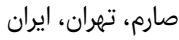

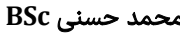
بخش ميكروبشناسى، مركز تحقيقات بارورى و نابارورى صارم، بيمارستان

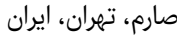

جكيده

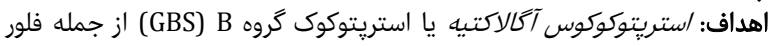

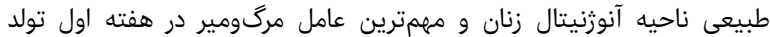

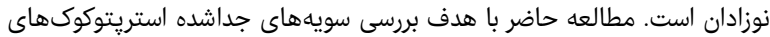
كروه B و الكوى مقاومت فنوتييى القاشده به كليندامايسين در زنان بدان باردار انجام

شد. - مون

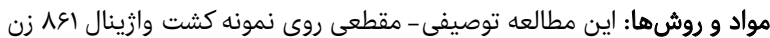

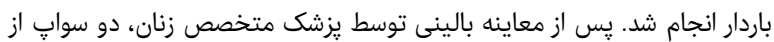

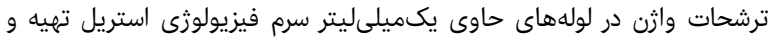

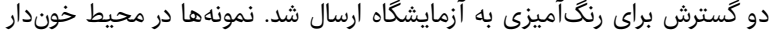

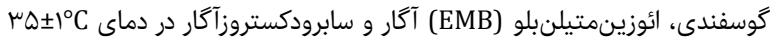

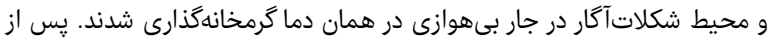

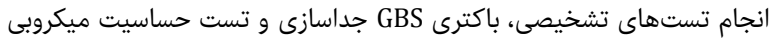

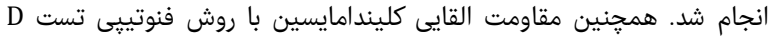

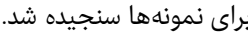

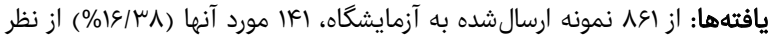

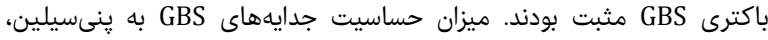

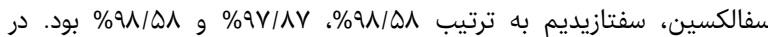

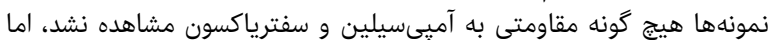

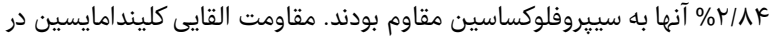

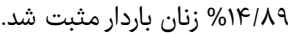

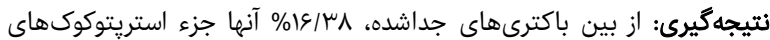

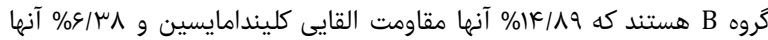

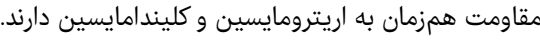

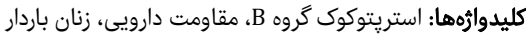

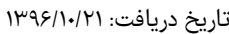

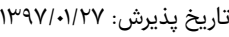
"نويسنده مسئول: nomanpoursh@gmail.com

مقدمه

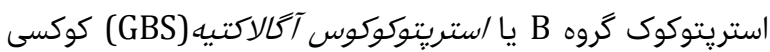

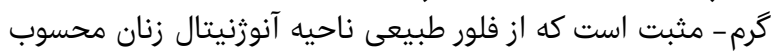

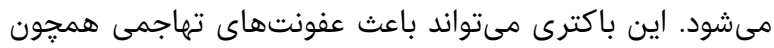

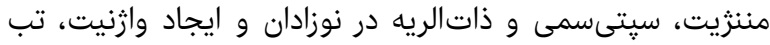

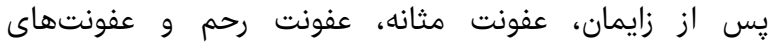

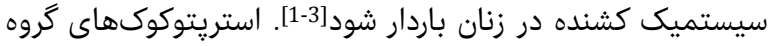

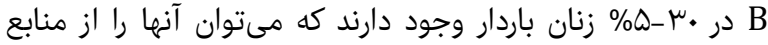

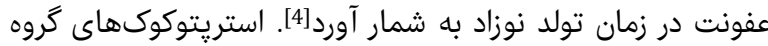

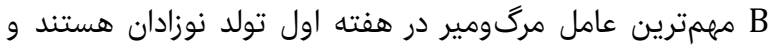

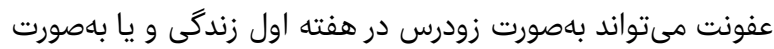

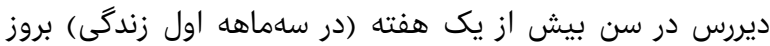




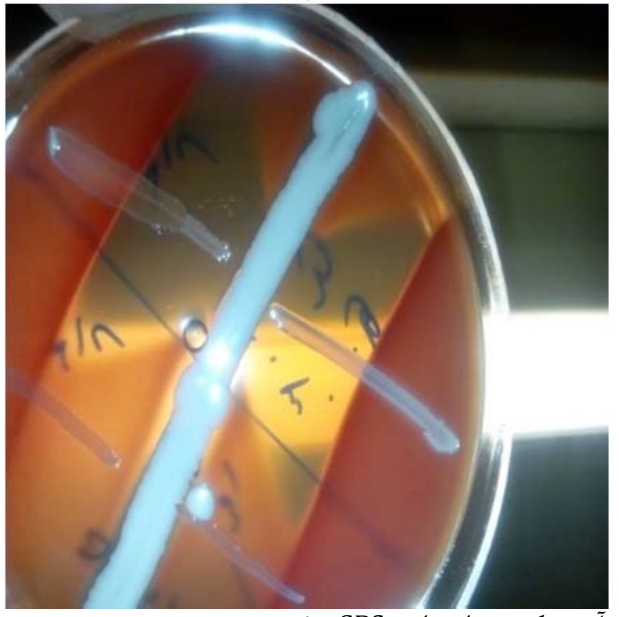

شكل ا) آزمون كمٍ براى تاييد GBS (أ| مورد)

جدول () ميزان حساسيت آنتىبيوتيكى (اعداد داخل يرانتز درصد هستند) مربوط به استريتوكوكنهاى جداشده (الئا مورد)

\begin{tabular}{|c|c|c|c|}
\hline مقاوم & نيمهحساس & حساس & آنتىبيوتيك \\
\hline$r(1 / \varepsilon r)$ & - & $1{ }^{\text {wq }}$ ( ( $\left.\Lambda / 0 \Lambda\right)$ & Pen \\
\hline $1(\cdot / V I)$ & $r(1 / \varepsilon r)$ & I $\wedge$ ( (qV/AV) & CN \\
\hline- & - & $|\varepsilon|(\mid \cdot \cdot)$ & CRO \\
\hline $1(\cdot / V \mid)$ & $1(\cdot / V I)$ & $\operatorname{lrq}^{\mu q}(9 \Lambda / 0 \Lambda)$ & CAZ \\
\hline- & - & $|\varepsilon|(\mid \cdot \cdot)$ & Amp \\
\hline$\varepsilon(r / \wedge \varepsilon)$ & - & $\mathbb{I}^{\mathrm{W} V}(9 \vee / / 7)$ & CP \\
\hline$\|(V / \Lambda)$ & $r \mu(1 T / \mu I)$ & I.V ( VO/A৭) & $\mathrm{CC}$ \\
\hline $\operatorname{rr}(10 / 7)$ & or $(\mu-/ \wedge \Lambda)$ & TV ( $(V / O r)$ & E \\
\hline
\end{tabular}

Pen

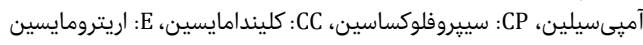

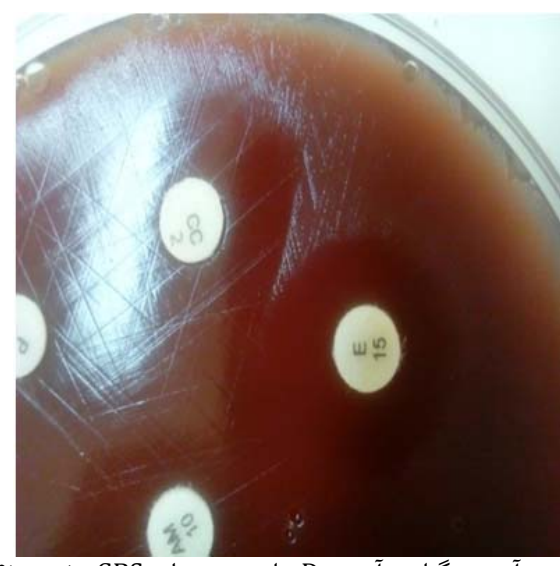

شكل r) آزمون آنتىبيوگرام و آزمون D براى نمونههاى GBS مثبت (اع| مورد)

بحث

استريتوكوى بتاهموليتيك گروه GBS) B) يكى از باكترىهاى

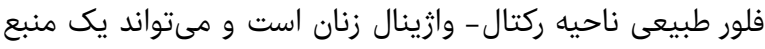

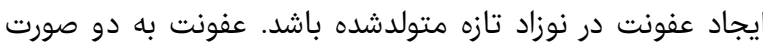

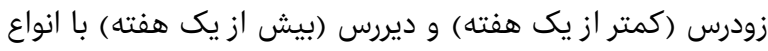

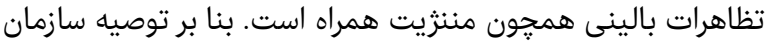

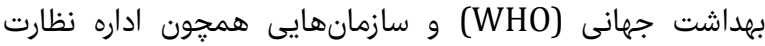

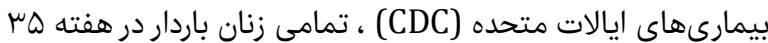

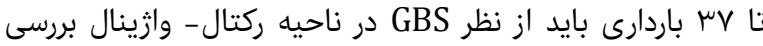

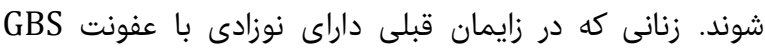

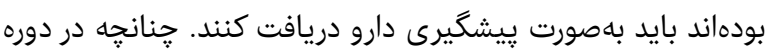
باردارى، كشت ادرار در زنان باردار از نظر بـاريد

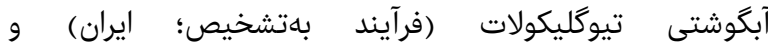

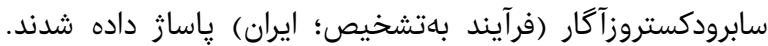

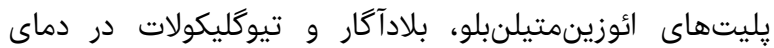

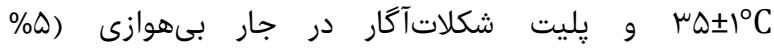

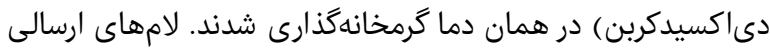

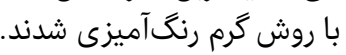

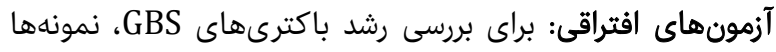

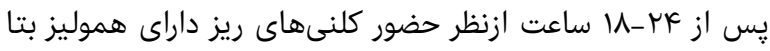

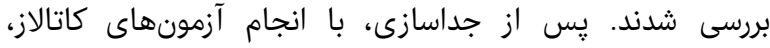

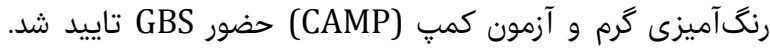

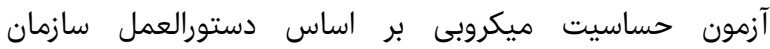

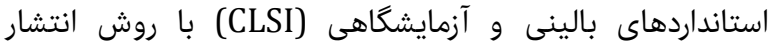

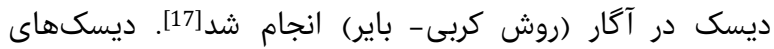

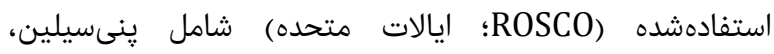

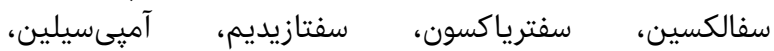

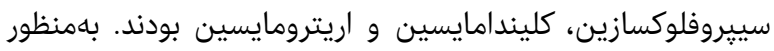

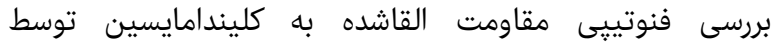

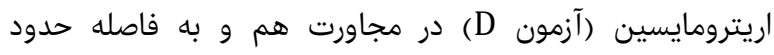

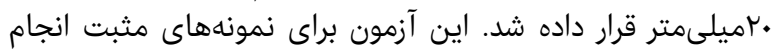

شد [4]. آناليز آمارى با استفاده از نرمافزار SPSS 20 انجام شد.

يافتهها

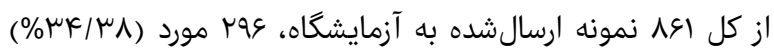

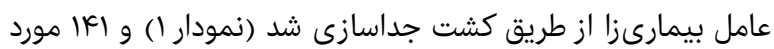

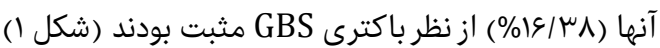

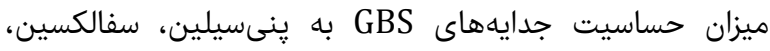

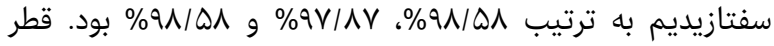

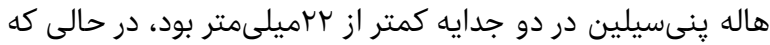

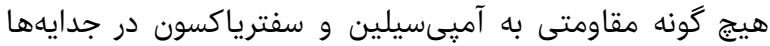

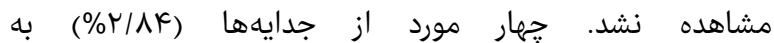

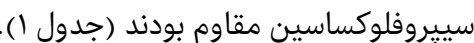

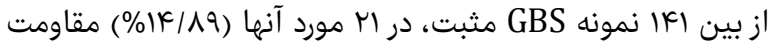

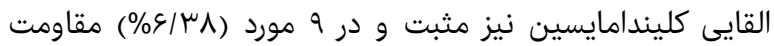

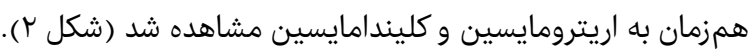

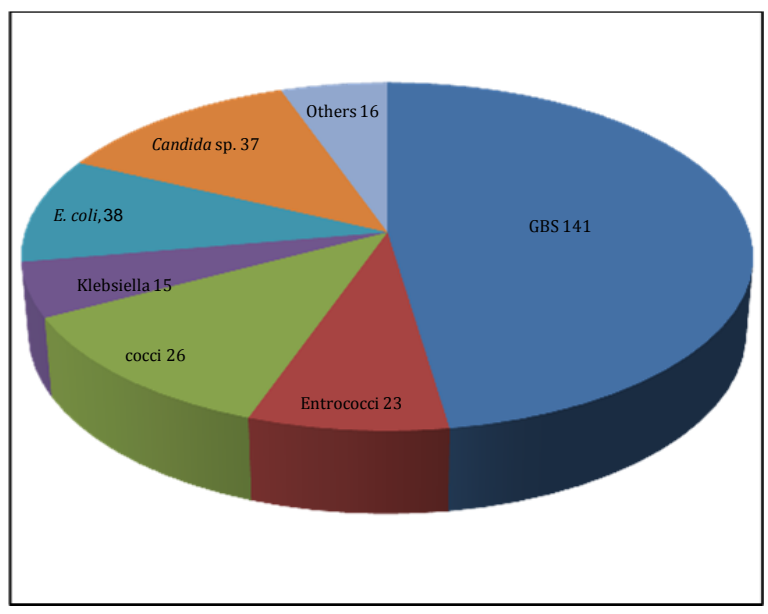

نمودار ا) توزيع فراوانى انواع باكترىهاى جداشده از نمونههاى ترشحات واثن

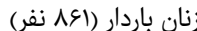

Volume 4, Issue 1, Spring 2019 
در ^ץ/\%\% آنها مقاومت همزمان به اريترومايسين و كليندامايسين

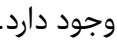

تشكر و قدردانى: جا دارد از همكاران محترم كلينيك يريناتولوزى

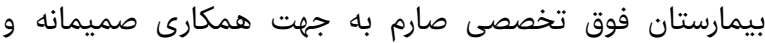

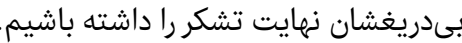

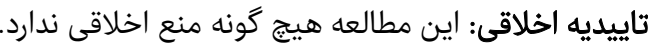

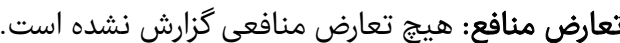

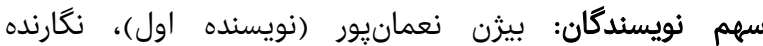

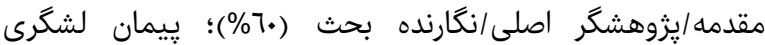

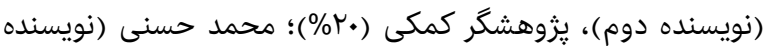

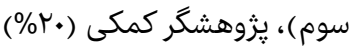

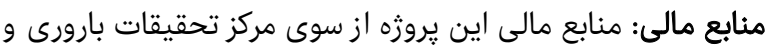
نابارورى صارم تامين گرديده ماست است

منابع

1- Leggat P. Therapeutic guidelines: Antibiotic. J Mil Veterans Health. 2010;18(4):36.

2- Andrews JI, Diekema DJ, Hunter SK, Rhomberg PR, Pfaller MA, Jones RN, et al. Group B Streptococci causing neonatal bloodstream infection: Antimicrobial susceptibility and serotyping results from SENTRY centers in the Western Hemisphere. Am J Obstet Gynecol. 2000;183(4):859-62.

3- Anthony BF. Carriage of group B Streptococci during pregnancy: A puzzler. J Infect Dis. 1982;145(6):789-93.

4- World Health Organization. WHO recommendations on interventions to improve preterm birth outcomes. Geneve: World Health Organization; 2015.

5- Baker CJ, Barrett FF. Transmission of group B Streptococci among parturient women and their neonates. J Pediatr. 1973;83(6):919-25.

6- Verani JR, McGee L, Schrag SJ. Prevention of perinatal Group B Streptococcal disease: Revised guidelines from CDC, 2010. Cent Dis Control Prev. 2010;59(RR10):1-32.

7- Bergseng $H$, Rygg $M$, Bevanger L, Bergh K. Invasive group B Streptococcus (GBS) disease in Norway 19962006. Eur J Clin Microbiol Infect Dis. 2008;27(12):11939.

8- Aali BS, Abdollahi H, Nakhaee N, Davazdahemami Z, Mehdizadeh A. The association of preterm labor with vaginal colonization of group B Streptococci. Int J Reprod Biomed. 2007;5(4):191-4.

9- Castor ML, Whitney CG, Como-Sabetti K, Facklam RR, Ferrieri P, Bartkus JM, et al. Antibiotic resistance patterns in invasive group B Streptococcal isolates. Infect Dis Obstet Gynecol. 2008;2008:727505.

10- Desjardins M, Delgaty K, Ramotar K, Seetaram C, Toye B. Prevalence and mechanisms of erythromycin resistance in group A and group B Streptococcus: Implications for reporting susceptibility results. J Clin Microbiol. 2004;42(12):5620-3.

11- Onipede AO, Onayade AA, Elusiyan JB, Obiajunwa PO, Ogundare EO, Olaniran 00 , et al. Invasive bacteria isolates from children with severe infections in a Nigerian hospital. J Infect Dev Ctries. 2009;3(6):429-36.

12- DiPersio LP, DiPersio JR. High rates of erythromycin and clindamycin resistance among OBGYN isolates of group B Streptococcus. Diagn Microbiol Infect Dis. 2006;54(1):79-82.

13- Kalantar E. High burden of group B Streptococcus: An invasive bacterium among pregnant women referring دومان بيزن نعمانيور و همكاران

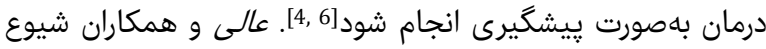
GBS

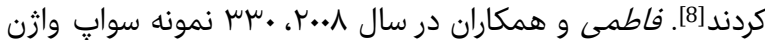

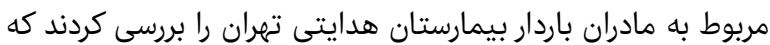

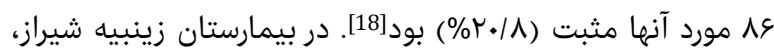

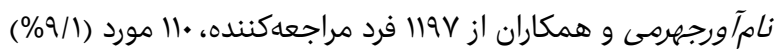

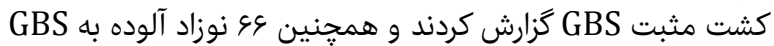

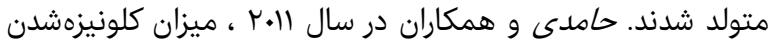
GBS

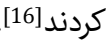

در مطالعات ذكرشده با توجه به شرايط اقليمى و جمعيتى ميزان

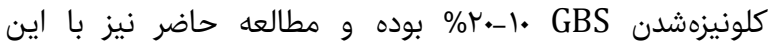

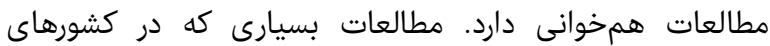

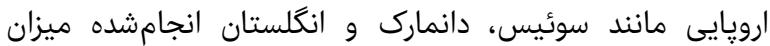

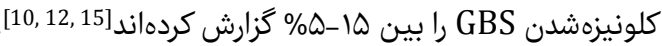

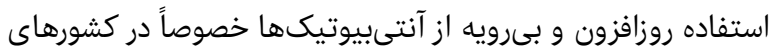

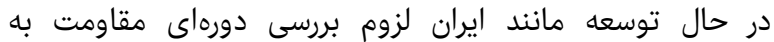

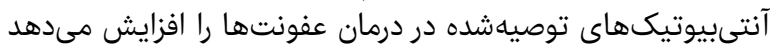

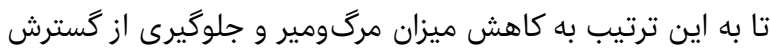

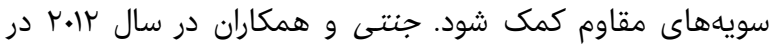
مطالعه هو جدايه GBS به اين نتيجه رسيدند كه تمامى نمون نمونه

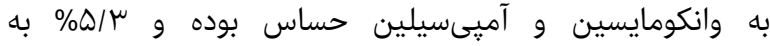

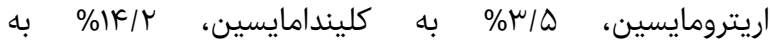

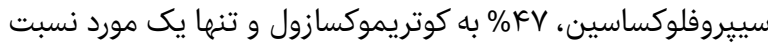
به ينىسيلين مقاوم بودند [19].

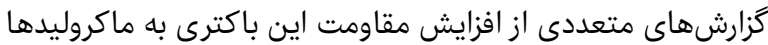

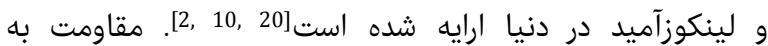

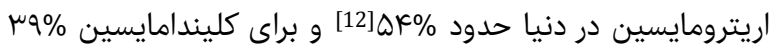

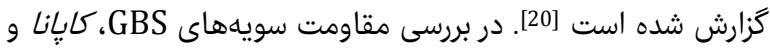

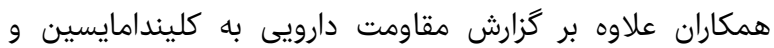

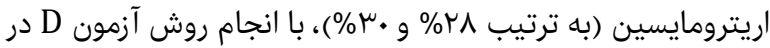

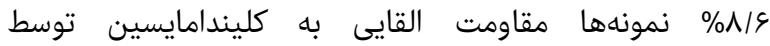
اريترومايسين نيز مشاهده كردند [17].

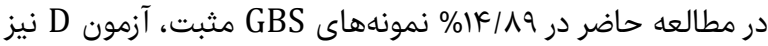

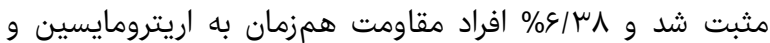

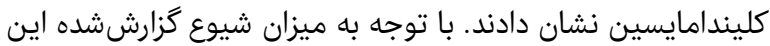

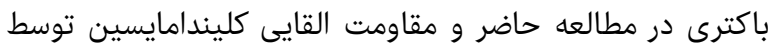

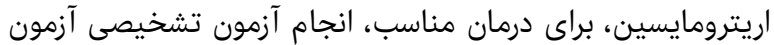

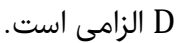

از محدوديتهاى اين مطالعه مى التوان به محدوديت در نمونهگيرى

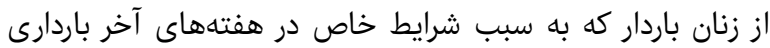

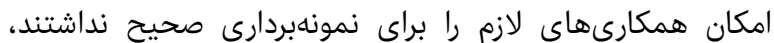

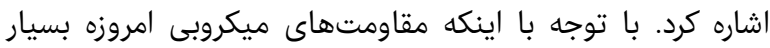

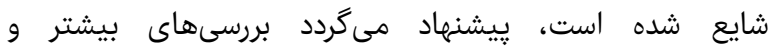

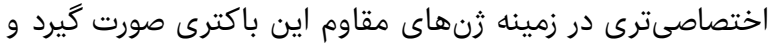

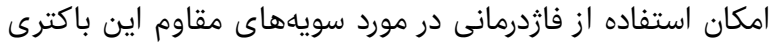
نيز مورد بررسى و مطالعات بيشتر قرار گيردان

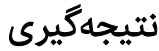

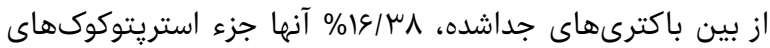

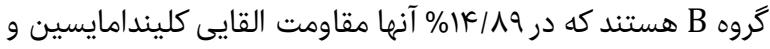




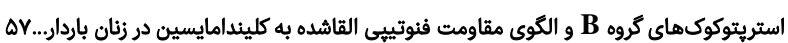
resistance patterns among group B Streptococcus isolates: Implications for antibiotic prophylaxis for earlyonset neonatal sepsis. Swiss Med Wkly. 2013;143:w13778.

18- Fatemi F, Chamani-Tabriz L, Pakzad P, Zeraati $H$ Rabbani H, Asgari S. Colonization rate of group B Streptococcus (GBS) in pregnant women using GBS agar medium. Acta Med Iran. 2009;47(1):25-30.

19- Jannati E, Roshani M, Arzanlou M, Habibzadeh S, Rahimi G, Shapuri R. Capsular serotype and antibiotic resistance of group B Streptococci isolated from pregnant women in Ardabil, Iran. Iran J Microbiol. 2012;4(3):130-5.

20- Janapatla RP, Ho YR, Yan JJ, Wu HM, Wu JJ. The prevalence of erythromycin resistance in group B Streptococcal isolates at a University Hospital in Taiwan. Microb Drug Resist. 2008;14(4):293-7. to health centers of Sanandaj, Iran. Infect Epidemiol Med. 2013;1(1):15-8.

14- Figueira-Coelho J, Ramirez M, Salgado MJ, MeloCristino J. Streptococcus agalactiae in a large Portuguese teaching hospital: Antimicrobial susceptibility, serotype distribution, and clonal analysis of macrolide-resistant isolates. Microb Drug Resist. 2004;10(1):31-6.

15- Barcaite E, Bartusevicius A, Tameliene R, Kliucinskas M, Maleckiene L, Nadisauskiene R. Prevalence of maternal group B Streptococcal colonisation in European countries. Acta Obstet Gynecol. 2008;87(3):260-71.

16- Hamedi A, Akhlaghi F, Seyedi SJ, Kharazmi A. Evaluation of group B Streptococci colonization rate in pregnant women and their newborn. Acta Med Iran. 2012;50(12):805-8.

17- Capanna F, Emonet SP, Cherkaoui A, Irion O, Schrenzel J, Martinez de Tejada Weber B. Antibiotic 